\title{
OBSERVATIONS OF LOOPS AND PROMINENCES
}

\author{
KEITH T. STRONG AND THE YOHKOH TEAM \\ Lockheed Solar \& Astrophysics Laboratory, Palo Alto, CA 94304, USA
}

\begin{abstract}
We review recent observations by the Yohkoh-SXT in collaboration with other spacccraft and ground-based observatories of coronal loops and prominences. These new results point to problems that SoHO will be able to address. With a unique combination of rapid-cadence digital imaging ( $\geq 32 \mathrm{~s}$ full-disk and $\geq 2 \mathrm{~s}$ partial-frame images), high spatial resolution ( $\geq 2.5$ arcsec pixels), high sensitivity $\left(\mathrm{EM} \leq 10^{42} \mathrm{~cm}^{-3}\right)$, a low-scatter mirror, and large dynamic range, SXT can observe a vast range of targets on the Sun. Over the first 21 months of Yohkoh operations, SXT has taken over one million images of the corona and so is building up an invaluable long-term database on the large-scale corona and loop geometry. The most striking thing about the SXT images is the range of loop sizes and shapes. The active regions are a bright tangle of magnetic field lines, surrounded by a network of large-scale quiet-Sun loops stretching over distances in excess of $10^{5} \mathrm{~km}$. The cross-section of most loops seems to be constant. Loops displaying significant $\Gamma$ 's are the exception, not the rule, implying the presence of widespread currents in the corona.

All magnetic structures show changes. Time scales range from seconds to months. The question of how these structures are formed, become filled with hot plasma, and are maintaincl is still open. While we see the propagation of brightenings along the length of active-region loops and in X-ray jets with velocities of several hundred $\mathrm{km} / \mathrm{s}$, much higher velocities are seen in the quiet Sun. In XBP flares, for example, velocities of over $1000 \mathrm{~km} / \mathrm{s}$ are common. Active-region loops seem to be in constant motion, moving slowly outward, carrying plasma with them. During flares, loops often produce localized brightenings at the base and later at the apex of the loop. Quiescent filaments and prominences have been observed regularly. Their coronal manifestation seems to be an extended arcade of loops overlying the filament. Reliable alignment of the ground-based data with the X-ray images make it possible to make a detailed intercomparison of the hot and cold plasma structures over extended periods. Hence we are able to follow the long-term evolution of these structures and see how they become destabilized and erupt.
\end{abstract}

Key words: Sun - Corona - Magnetic Structure

\section{Introduction}

The recent flood of results from Yohkoh on the nature of coronal structures is rhanging our vicw of the solar atmosphere. The picture that is emerging is one of an extremely dynamic and complex corona. The high-cadence imaging available from the Soft X-ray Telescope (SXT) on Yohkoh makes it possible for the first time to observe the detailed evolution of coronal structures. These data are leading to new questions about how the corona is healed and supplied with mass, how active regions emerge and evolve, and how flares are produced. Iowever, perhaps the most surprising aspect of these data is how they are causing us to reevaluate the basic building block of coronal structure, the magnetic loop. Coronal loops are not the stable, scmicircular, potential structures that are often used in models. Rather, they seem to be asymmetric and dynamic structures with their own complex internal structure. This poses a problem that cannot be solved with 
existing data, namely that the processes that produce a system of loops are occurring on rapid timescales and over a broad range of temperatures. So to understand this complex structure, we will need to adopt a multiwavelength approach. SoHO, with supporting observations, offers the best opportunity to probe the magnetic structure of the solar atmosphere at different heights simultaneously with high-resolution imaging and a wide range of plasma diagnostics.

In this paper, we illustrate the level of complexity and the range of geometries of coronal loops. Then we will show some of the advantages of the multiwavelength approach that SoHO will be able to take, especially in conjunction with other space-based missions such as Yohkoh and with the network of ground-based instrumentation. To do this we will discuss joint observations between Yohkoh and several recent rocket-borne payloads that observed filaments.

\section{Coronal Currents}

When we look at a full-disk image from SXT, the striking impression is that there is a wide variety of loops. In Figure 1 we can see some postflare loops on the northwest limb (upper right). Several bright and complex active regions show a tortured weave of loops. The quiet-Sun loops tend to be larger, fainter, and low-lying. X-ray bright points (XBPs) are tiny, shortlived bipolar regions. They seem to be smaller versions of active regions, but when examined closely, they are found to fluctuate and flare (Strong et al. 1992 ). One of the first things noticed about the SXT images were that the loops seemed to be uniform in cross-section. This discovery was quantified by Klimchuk et al. (1992), who showed that the upper limit on the $\Gamma$ of the loop was about 1.1. They attributed this effect to currents flowing along the loops, producing a toroidal ficld which maintains a near-constant crosssection. This result is in direct contrast to the submerged dipole model of coronal loops which came out of the Skylab era.

This interesting result implies the presence of coronal currents, whose dissipation has long been proposed as a source of coronal heating. This conclusion is supported by Lang et al. (1993), who used an entirely independent method. They compared SXT images with VIA and R. IT $\backslash N-500$ radio maps and were able to calculate the coronal magnetic field strength and compare it with the extrapolated potential field determined from a magnelogram. The measured coronal fields were significantly stronger than predicted by the model, indicating that the divergence of the field was less than expected.

These results imply that coronal currents are a significant lactor in determining the geometry of the loops; however, that is not proof that they have a significant role in heating the corona. If that were the case, we would expect that the brightest and hottest coronal loops would be well correlated with

yohkoh.tex - Date: January 12, 1994 Time: 10:36 
the strongest currents in the corona.

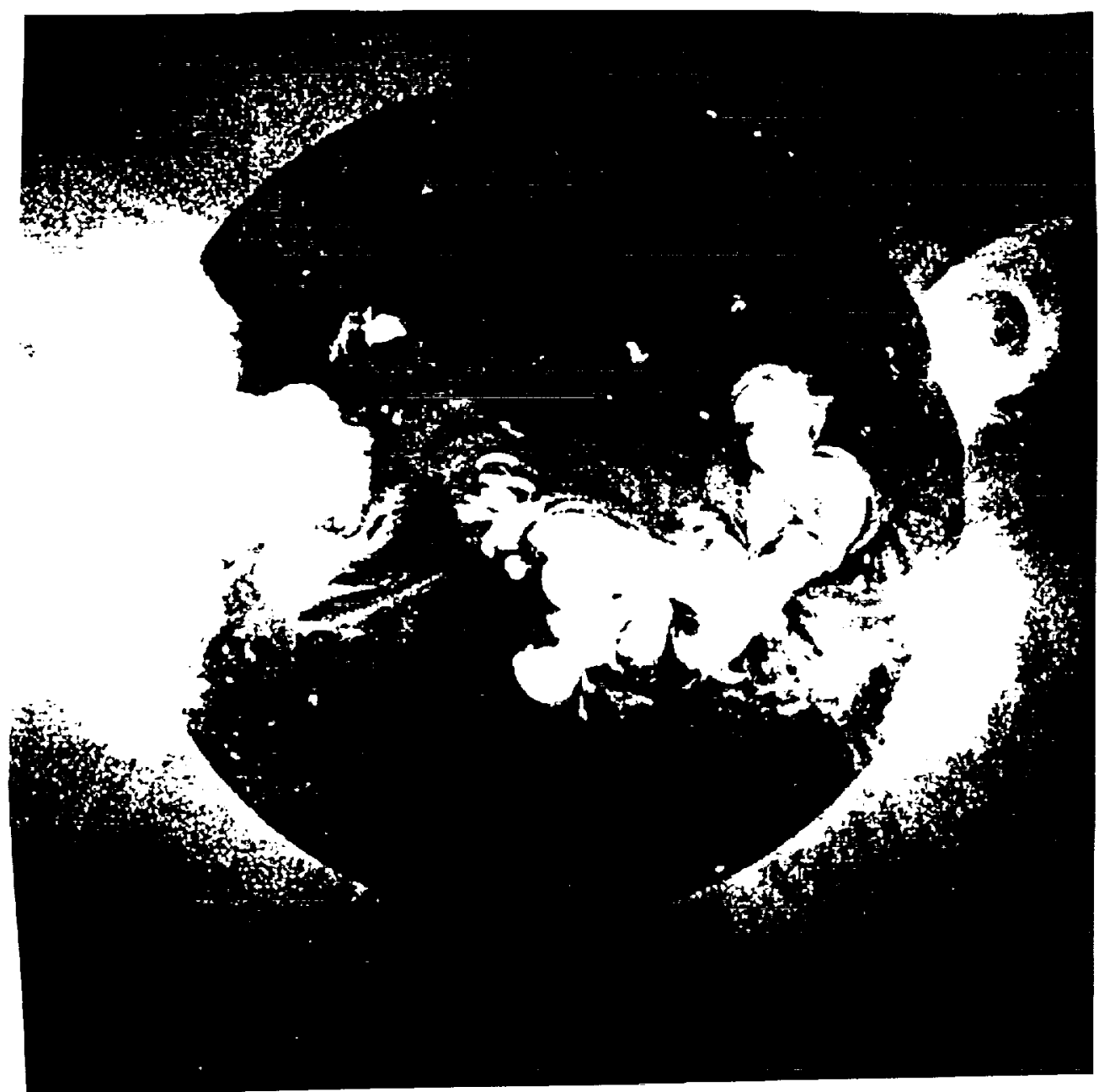

Fig. 1. SXT image of the corona (log scaling of a 2.536-s exposure taken iu the Ml 1400 filter).

Metcalf ot al. (1993) have found a way of checking this hypollessis by using SXT data in conjunction with Mees vector magnetograms. From these data they can flerive the location and strength of the coronal currents and find no significant correlation with bright, soft $\mathrm{X}$-ray loops. At first glance this secms to eliminate the possibility of currents heating the corona. It does eliminate coronal heating by large-scale, organized current systems. Ilowever, there still mmains the possibility that a chaotic, randomly directed system of filamentary currents exists in the corona and heats it. 


\section{Loop Geometry}

There are other indications that currents exist in the corona and play a significant role in determining loop geometry. Evidence for currents can be found in the existence of sheared and twisted loops. While sheared loops abound in SXT images, remaining stable for hours to days, twisted loops are quite rare and scem to be unstable. Figure 2 shows one of the clearest examples of the development of a twisted loop.

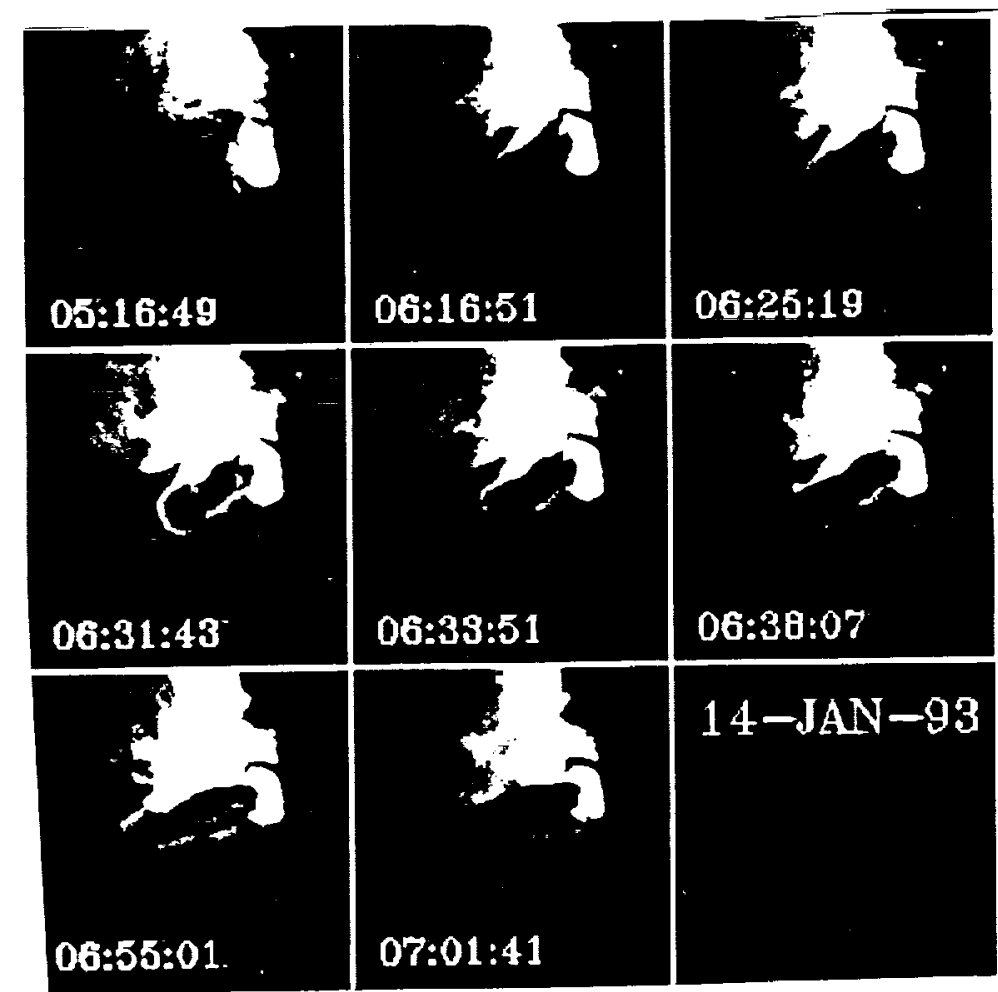

Fig. 2. The development of a possible kink instability in a loop on the southeast limb. Initially this loop has an ord angular shape, but it slowly develops over the next 20 min to a more smootli, curved shape. Sucklenly a kink clearly forms in the northeast corner of the loop, after which it rapidly, almost explosively, expands and dissipates. Note the fine twisted structure of the fied revealed as the loop expands.

One type of loop geometry is much more common in the Yohkoh images than we expected, and that is the cusped loop. Wo see many different sizes of cusped loop, ranging from a few arcseconds to over a solar radius. Several examples are shown in Figure 3. The cusped loops observed by Yolkoh associated with coronal mass ejections (CMEs) and flares are also discussed clscwhere in this volume (see Iliej ot al. 1994 and Cheng et al. 1994, respectively). Both of these types of cusped loops sem to be associated with an 
arcade of loops that can stretch over several arcmin.

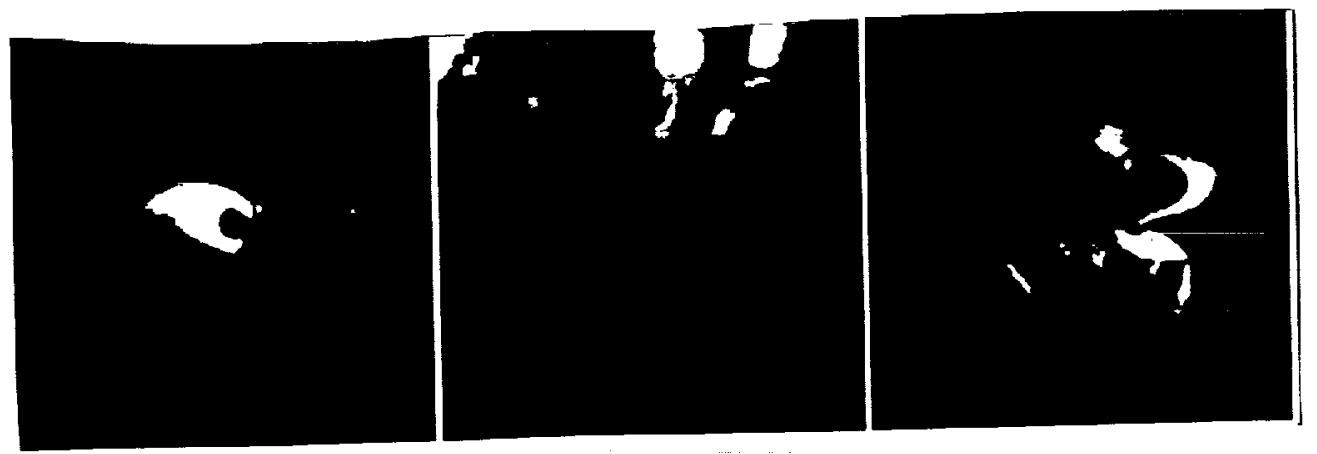

Fig. 3. Examples of cusped loops from flares (left), flament eruptions (centre), and active regions (right).

The small, bright cusped loops particularly associated with long-duration flares are dynamic and last for a few hours. They grow at a rate of 5 to 10 $\mathrm{km} / \mathrm{s}$ and generally have a bright apex (Figure $3 \mathrm{a}$ ). The Yohkoh data on these seem to be consistent with the idea that these structures are formed by the successive reconnection of open field lines higher and higher in the corona. The larger-scale cusped loops are very reminiscent of helmet streamers and also expand upwards slowly (Figure 3b). They often seem to be associated with the aftermath of a flament eruption. They can last for several days and are often explained in similar terms to the smaller, flare-related structures. However, there are some important differences. They are very rare; we have observed only about 10 such events clearly in over two years. CMEs and filament eruptions are far more common than these large-scale cusped loops. Unlike their flare-related cousins, they are rarely bright at the apex of the helmet, implying that reconnection is not going on there, if it were, one would expect a somewhat elevated temperature due to local heating, which would produce a significant increase in the brightness at the apex. The explanation of these events by the reconnection of open fields was needed the explain the slow growth of the helmet. However, other loops have been seen to grow through the same region prior to the event. None of these had a cusp-like structure. Further, we observe expanding loops in active regions (Uchicla et al. 1992) that are not necessarily explained in terms of reconnection as the driving mechanisim (although it might be caused by the expanding loops interacting with existing field lines). Although none of these objections to the current reconnection models for large scale X-ray helmet streamers are in and of themselves a fundamental problem, they should make us pause to rexamine our assumptions about the mechanism that forms these structures.

yohkoh.tex - Date: January 12, 1994 Time: 10:36 
There is another type of cusped loop often seen in the quiet Sun or in active regions. These share the general geometry but seem to have a fundamentally different structure (Figure 3c). They seem to be thin structures, i.e., not associated with an arcade of loops. They do not seem to grow rapidly but can last from a few minutes to over a day. One of their most surprising aspects is that they can often be seen to be reconnected back to the Sun via a fine loop-like structure to another cusped loop. If this is the actual structure of these loops, then the filamentary loop joining the two cusps is probably a current sheet. This leads one to speculate how many of the other loop-like structures we see in the corona are in fact current sheets. The answer seems to be not many, but we should be careful when interpreting the properties of any given loop, because it might be a current sheet.

So far, Yohkoh is providing us with a complex picture of the solar corona. It is highly dynamic, changing on time scales from seconds to days. These changing loop structures have spatial scales from the practical limit of the instrument $(5$ arcsec) up to about a solar radius. The complexity of the geometry that is observed is often daunting.

\section{The Multiwavelength Solution: SoHO}

The real problem with interpreting the SXT data lies in that there is no information about the cooler $(\leq 2 \mathrm{MK})$ plasma and that the continuity of the data is broken by regular, long periods of spacecraft night. Sollo will remove these limitations. To illustrate this point, we shall describe collaborative observations of filaments and prominences in this section.

\section{Prominences and Overlying Coronal Arches}

In Figure t we show simultaneous observations by SXT, the TransitionRegion Camera (TRC) on the SPDE rocket, and 10830 A images (KPNO) of a filament channel. Note that in the (' IV image, several clear, datk channels run across the image where the bright network structure is suppressed. Comparing this with the $10830 X$ image, we can identify arcas of dark filaments, but they exist only along portions of cach channel. It is clear that the coronal loops are most sheared along (i.e. parallel to) the filament channel where the rlarkest and most prominent filaments can be secn. There is little evidence of any overlying magnetic ficld lines in Figure t.

To observe these overlying loops more clearly, we have to look all prominences. Figure 5 shows a set of images from NIXT, BBSO, and Yolıkoh, taken just after the SPARTAN flight on 13 April 1993. I prominence can be secn in the II image on the southeast limb. The same prominenen is clearly visible in absorption in the Fo XII and Fo XV images from NIXT'. Such absorption fatures are not usually seen in coronal images, but as. NIXT

yohkoh.tex - Date: January 12, 1994 Time: 10:36 
operates in the XUV region of the spectrum, the light is more easily absorbed than at soft $\mathrm{X}$-rays wavelengths. The SXT image shows a dark cavity where the prominence is situated, but a faint arch of overlying magnetic field lines can be seen above the cavity. These two data sets seem very reminiscent of the model discussed by Antiochos et al. (1994) at this conference, in which highly sheared field lines along the neutral line support the filament material and overlying (near potential) fields confine it.

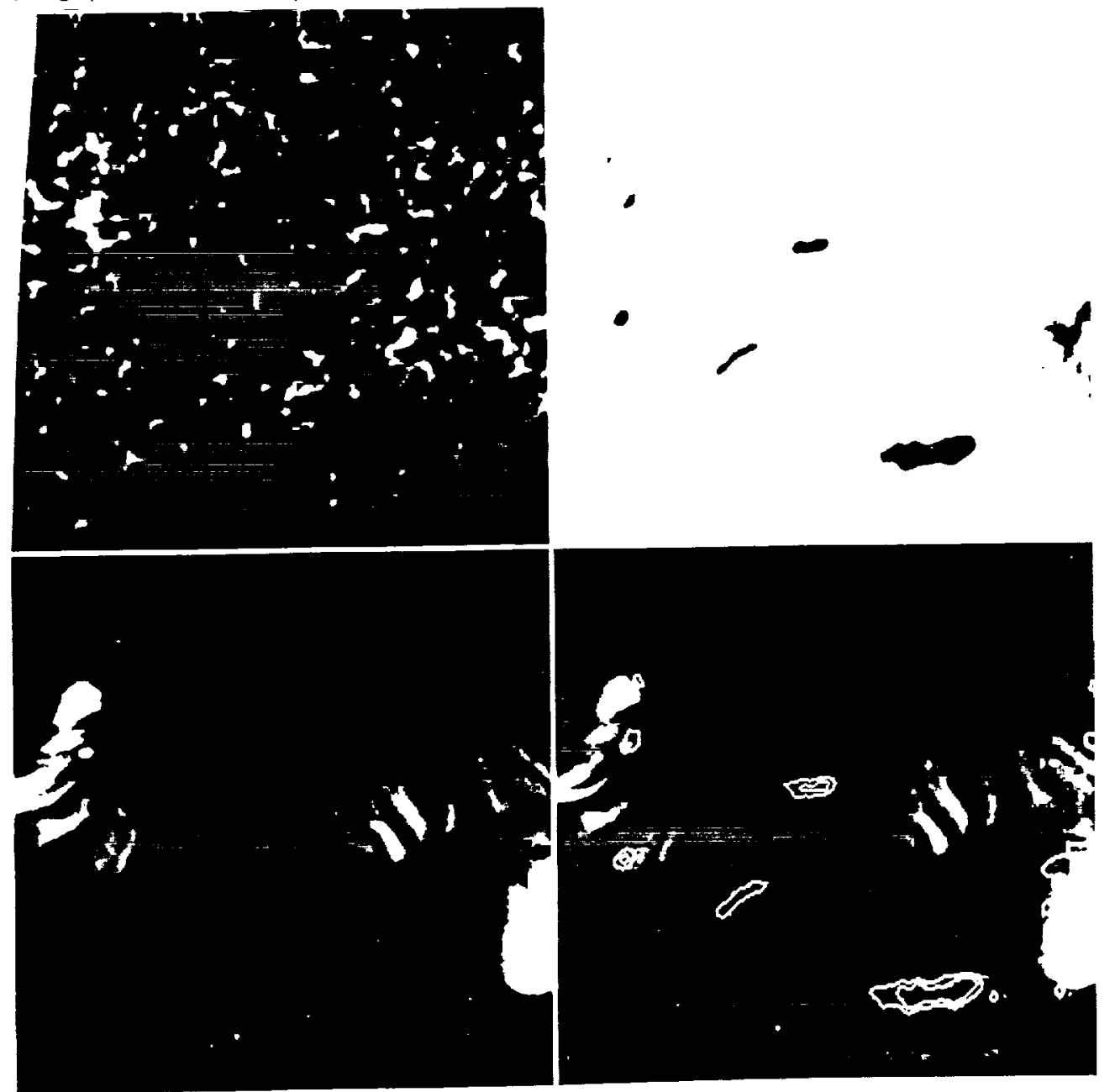

Fig. 4. Comparison of UV (C IV from SPDE), $10830 \lambda$, and SXT images of filament channels.

These data illustrate the structure of a filament in the corona. What happens on a larger scale? We need to compare images from the lower corona with data from white-light coronagraphs. The only large-scale coronagraph observations that have been available during Yohkoh have been those from 
the recent SPARTAN mission. Figure 6 shows a composite SPARTAN and SXT image from 12 April 1993.

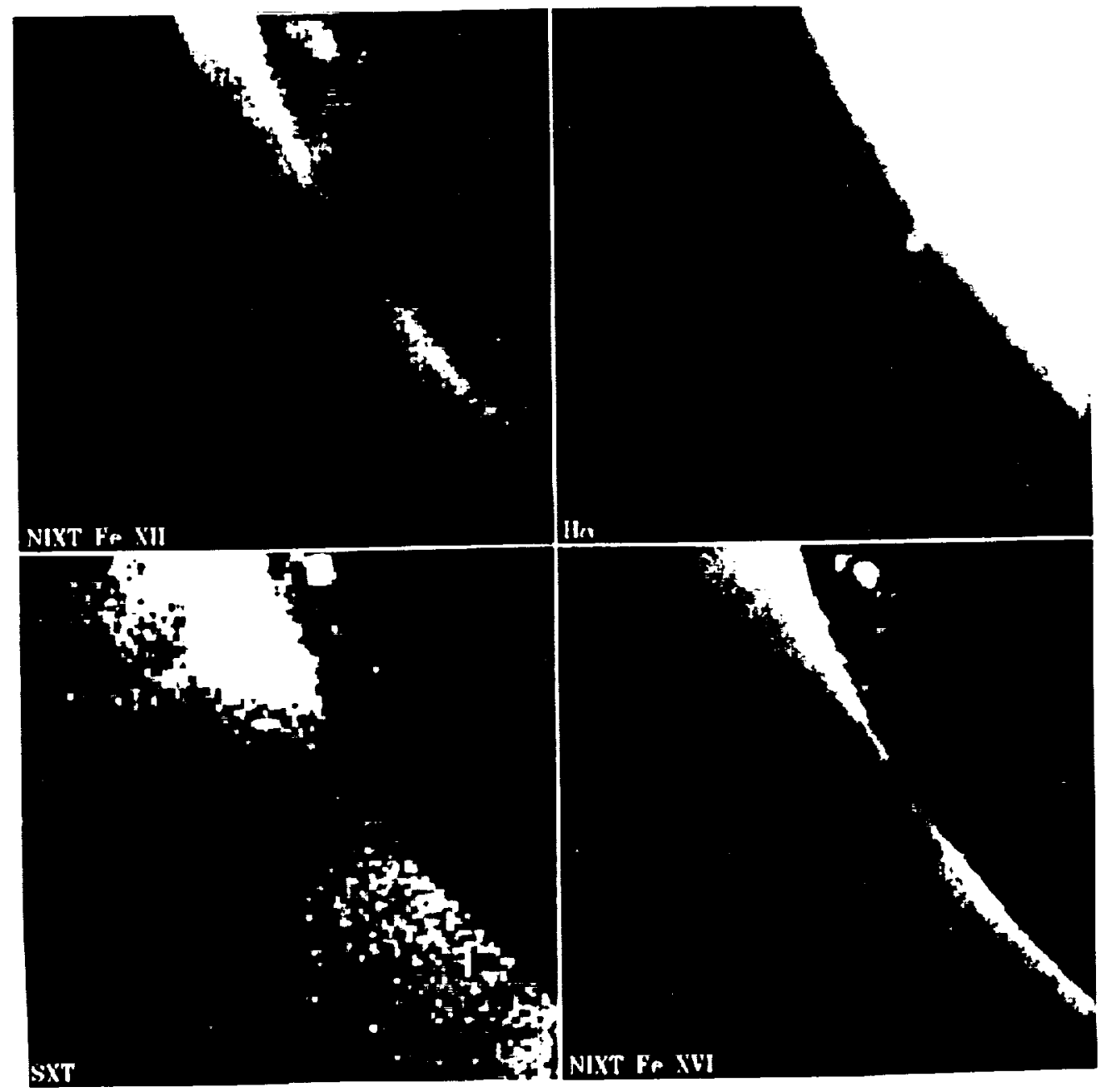

Fig. 5. Comparison of IIo (BBSO), XUV (Fe XII and Fe XV from NIXT, courtesy L. (iolub), and SXT images of a prominence on the SE limb.

Note that there are three prominent helmet streamers visible: two along the cquator of the Sun and one in the southeast. Of the two equatorial helmet stremers, the one on the west limb is by far the brighter, probably because of a coronal transient and flare from the western active region below it. In fact, an arcade of postlare loops can be seen about 5 arcmin above the west limb. However, the brightest and most prominent helmet does not lie above the active regions but, rather, over the filament channel on the southeast, which is the same one as shown in Figure 5. So, as SoHO will be able to do with its unique combination of instruments, we have been able to 
investigate the magnetic structure of a prominence from the chromosphere out to several solar radii.

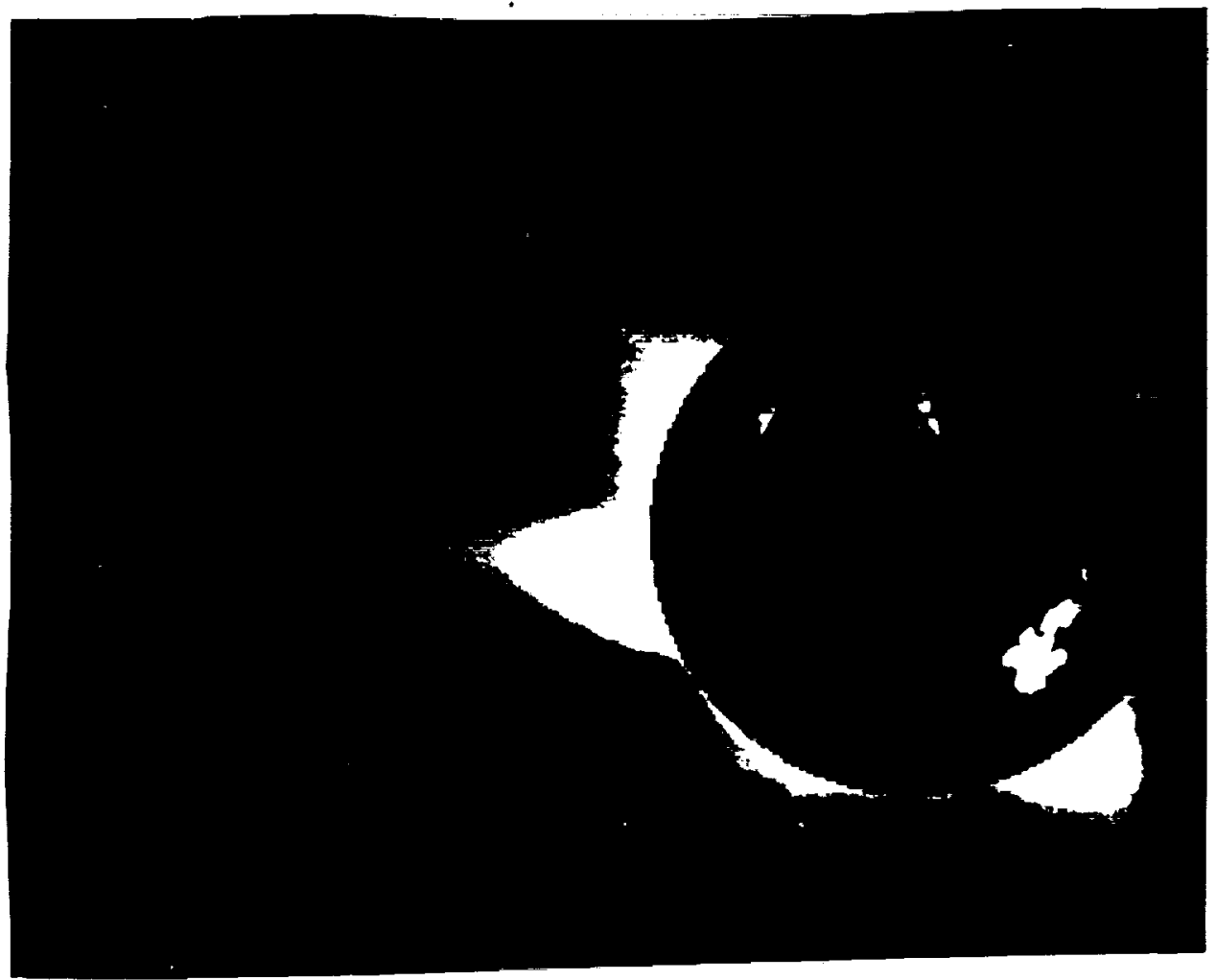

Fig. 6. A white light coronagraph image (SPARTAN) of the streamer above the prominence shown in Figure 5, taken on 12 April 1993.

\section{Conclusions}

All magnetic structures are seen by SXT to change on time scales that range from seconds to montlis. While we see the propagation of brightenings are seen along the length of active-region loops and in $\mathrm{X}$-ray jets with velocities of several hundred $\mathrm{km} / \mathrm{s}$, much higher velocities are seen in the quiet Sun. In XBP flares, for example, velocities of over $1000 \mathrm{~km} / \mathrm{s}$ have been observed. Active-region loops secm to be in constant motion, moving slowly outward, carrying plasma with them. During flares, loops often produce localized brightenings at the base and later at the apex of the loop. Hence Yohkoh is presenting a confused and complex picture of the solar atmosphere, which may have to wait for observations from SollO to be understood better.

Sollo will be able to look much further out into the corona than the above Yohkoh observations and have available UV and EUV spectral diag- 
nostics. It has uninterrupted viewing of the corona and so should be able to clearly see the formation, development, and decay of most solar structures. Hence we look forward to SoHO to shed further light on the many problems associated with the magnetic structure of the solar atmosphere and its defining emission.

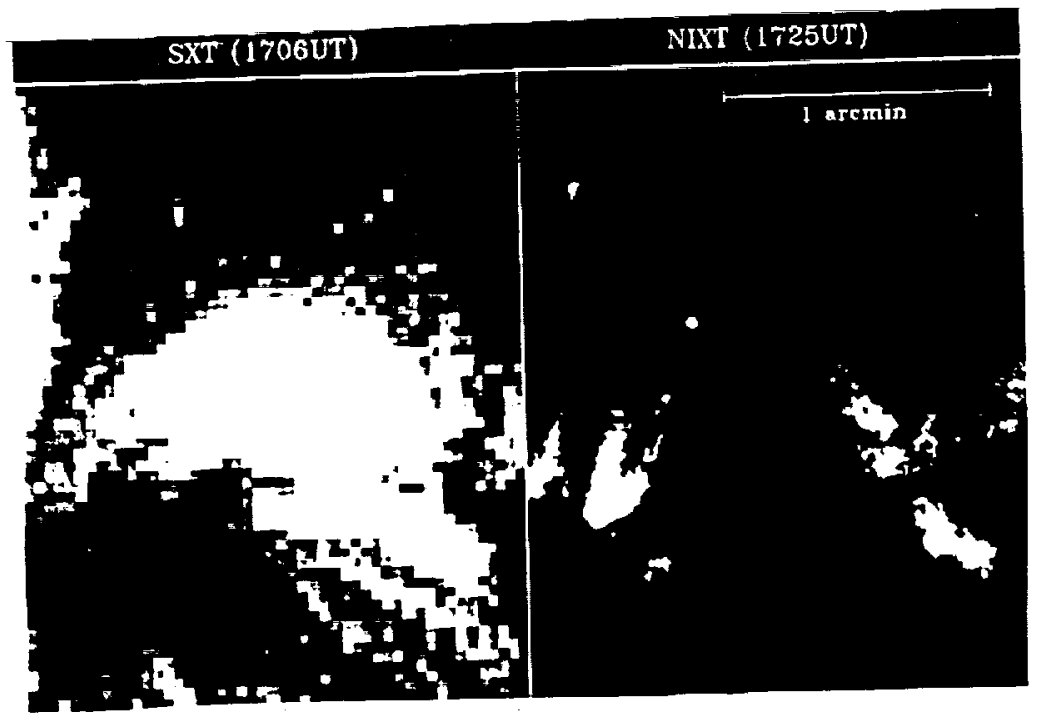

Fig. 7. Comparison of broad-band (left) and multilayer (right) images of quiescent loops. Comparison of observations of a quiescent active region seen through SXT and NIXT (courtesy L. Golub).

The ideal situation would be to have Yohkoh still operating during SoHO, because its observations will complement those of SoHO, not only from the differences in the response of a broarl-band $\mathrm{X}$-ray telescope compared with multilayer optics (Figure 7) but also in the sense that the EIT full-disk images will be very limited in number owing to telemetry limitations of SoIIO. SXT obtains several hundred full-clisk images each day and thousands of partial-frame images. All of the Yohkoh instruments can respond to flares which inay be beyond the saturation limit of some of the SoHO instruments, and so Yohkoh can provide information of any events that might affect SOIIO. Hence SoIIO, Yohkoh, and TRACE (Strong et al. 1994) will provide a powerful set of imaging and spectroscopic tools that should be able to answer many of the questions that have perplexed us for so many years.

\section{Acknowledgements}

This work is supported by NASA contract NAS8 32334 with MSFC and the Lockheed Independant Research Programme. Yohkoh is operated at IS $\Lambda S$ by a team of Japanese, Mmerican, and British scientists whose dedication has 
made these data available to the solar community. SXT was built at Lockheed Solar and Astrophysics Laboratory in collaboration with the National Astronomical Observatory of Japan, the University of Tokyo, and ISAS

\section{References}

Antiochos, S. et al.: 1994, this volume, Cheng, C.-C. et al.: 1994, this volume, Hiei, E. et al.: 1994, this volume, Klimchuk, J. et al.: 1992, PSAJ 44, L181 Lang, K. et al.: 1993, $A p J$ 419, 298 Netcalf, T. et al.: 1994, $A p J$ in press, Strong, K.T. et at.: 1992, PSAJ 44, L161 Strong, K.T. et at.: 1994, this volume, Uchida, Y. et al.: 1992, PSAJ 44, L155 
